\title{
Trocas Afetivas Mãe-bebê: Revisão Integrativa da Literatura
}

\author{
Clarice Bieler* \\ Universidade do Estado do Rio de Janeiro - UERJ, Rio de Janeiro, RJ, Brasil \\ ORCID: http://orcid.org/0000-0003-4853-7627 \\ Deise Maria Leal Fernandes Mendes** \\ Universidade do Estado do Rio de Janeiro - UERJ, Rio de Janeiro, RJ, Brasil \\ ORCID: http://orcid.org/0000-0003-3487-7284
}

\begin{abstract}
RESUMO
Bebês buscam ativamente interações sociais, favorecendo trocas afetivas consideradas essenciais ao desenvolvimento. Investigações a respeito propiciam maior compreensão do processo de desenvolvimento emocional infantil. O objetivo deste estudo foi traçar um panorama atual acerca das trocas afetivas mãe-bebê, examinando características das publicações científicas nessa temática entre 2008 e 2018. Realizou-se uma revisão integrativa da literatura que permite uma síntese de conhecimentos a respeito, tendo-se empreendido uma busca nas bases de dados Web of Science, PsycNet, PUBMED, Scielo e LILACS. Foram selecionados 17 artigos para análise, após aplicação de critérios de inclusão e exclusão, sendo pré-estabelecidas categorias para analisar os estudos. Os resultados indicaram baixo número de publicações sobre o tema no período considerado (sendo 2018 o ano com maior número), predomínio de estudos transversais, em grandes centros urbanos, com crianças de zero a 36 meses. A temática geral mais abordada foi a da dinâmica e circunstâncias das trocas afetivas iniciais. Foi possível apontar lacunas quanto à necessidade de estudos voltados para a diversidade cultural, contemplando diferença de sexo dos bebês e que incluam microanálises de comportamentos afetivos e turnos nas trocas afetivas. Argumenta-se ainda a necessidade de novos estudos brasileiros em que se explorem contextos socioeconômicos diversos.
\end{abstract}

Palavras-chave: trocas afetivas, interações mãe-bebê, interações afetivas iniciais.

\section{Mother-Baby Affective Exchanges: Integrative Literature Review}

\begin{abstract}
Babies actively seek social interactions, favoring affective exchanges considered essential for development. Investigations about this provide a better understanding of the process of the child emotional development. This study aimed to draw a current panorama about the motherbaby affective exchanges, examining characteristics of scientific publications on this theme, between 2008 and 2018. An integrative literature review was carried out, allowing a synthesis of knowledge about it. A search was made in the Web of Science, PsycNet, PUBMED, Scielo and LILACS databases. Seventeen articles were selected for analysis, after applying inclusion and exclusion criteria, and categories were pre-established to analyze the studies. The results indicated a low number of publications on the subject in the considered period (2018 being
\end{abstract}

ISSN $1808-4281$ 
the year with the largest number), predominance of cross-sectional studies in large urban centers, with children from zero to 36 months old. The most addressed general theme was the dynamics and circumstances of the initial affective exchanges. It was possible to point out gaps regarding the need for studies focused on cultural diversity, including gender differences in babies and including microanalysis of affective behaviors and shifts in affective exchanges. It is also argued the need for new Brazilian studies exploring different socioeconomic contexts.

Keywords: affective exchanges, mother-baby interactions, initial affective interactions.

\section{Intercambios Afectivos Madre-bebé: Revisión Bibliográfica Integradora}

\section{RESUMEN}

Bebés buscan activamente las interacciones sociales, favoreciendo intercambios afectivos considerados esenciales para el desarrollo. Investigaciones sobre esto proporcionan una mejor comprensión del proceso de desarrollo emocional del niño. El objetivo de este estudio fue dibujar un panorama actual sobre intercambios afectivos entre madre y bebé, examinando las características de publicaciones científicas sobre este tema (2008 a 2018). Se realizó una revisión integradora, que permitió una síntesis del conocimiento al respecto, buscando en las bases de datos Web of Science, PsycNet, PUBMED, Scielo y LILACS. Diecisiete artículos fueron seleccionados para análisis, después de aplicar criterios de inclusión y exclusión. Categorías fueron preestablecidas para el análisis. Resultados indicaron bajo número de publicaciones sobre el tema en el período considerado (2018, año con el mayor número), predominio de estudios transversales en grandes centros urbanos, con niños de cero a 36 meses. El tema general más abordado fue la dinámica y las circunstancias de intercambios afectivos iniciales. Fue posible señalar lagunas con respecto a necesidad de estudios centrados en la diversidad cultural, incluidas las diferencias de género en los bebés y el microanálisis de comportamientos afectivos y cambios en intercambios afectivos. Se argumenta la necesidad de nuevos estudios brasileños que exploren diferentes contextos socioeconómicos.

Palabras clave: intercambios afectivos, interacciones madre-bebé, interacciones afectivas iniciales.

A relevância conferida por estudiosos da psicologia do desenvolvimento às interações iniciais mãe-bebê, como contexto privilegiado de desenvolvimento, parece inconteste. Tal relevância também se aplica, particularmente, ao domínio do desenvolvimento emocional e afetivo. Neste campo, pode ser observado interesse crescente de pesquisadores quanto a diferentes aspectos e dimensões deste processo. Nas etapas iniciais, pelas características biopsicossociais presentes e pelas possibilidades de conquistas e avanços que envolvem, investigações científicas representam oportunidades de aprofundamento do conhecimento a 
respeito de como os indivíduos começam a experimentar e lidar com emoções e afeto em suas vidas e a interagir socialmente.

A literatura sobre o desenvolvimento inicial traz evidências quanto a capacidades associadas à interação social apresentada pelos bebês ao nascerem, a partir de predisposições vistas como produtos do processo evolucionário da espécie humana (Seidl-de-Moura \& Ribas, 2004). O bebê, segundo essa literatura, apresenta um conjunto de traços fisionômicos infantis que o tornam atraente e com aspecto de desamparo, compondo recursos que atraem cuidadores e provocam predisposições destes para cuidar, o que configura um cenário propício a trocas afetivas. Diversas habilidades e capacidades presentes logo ao nascimento, ou desde muito cedo, vêm sendo, há décadas, relatadas nos estudos, incluindo a discriminação e a preferência pela voz da mãe em relação a de outra mulher e a preferência por faces humanas (Walton, Bower, \& Bower, 1992). Evidências também apontam a percepção progressiva das expressões faciais dos outros (Thompson, 2015), a capacidade de produzir expressões faciais e de chamar a atenção de cuidadores (Cole \& Moore, 2014) e o choro como propiciadores de interações recíprocas e reguladores das trocas iniciais (Seidl-de-Moura \& Ribas, 2004).

A ação recíproca nas interações iniciais mãe-bebê e seus significados passaram a ser reconhecidos em estudos científicos, sobretudo, a partir da década de 1980. As capacidades da mãe e do bebê, suas ações e reações, com ênfase na reciprocidade entre parceiros, assim como a participação ativa do bebê nas trocas com adultos, passaram a ser investigadas a partir desse período, de acordo com a revisão de Seidl-de-Moura e Ribas (2004).

O segundo mês de vida tem sido visto como um momento de importantes mudanças quanto à sensibilidade para demonstrações de afeto e para respostas contingentes às expressões emocionais dos cuidadores. Autores como Rochat e Striano (2010) indicam que aos dois meses há um crescimento da quantidade de tempo despendido em estados de alerta, mudanças quanto ao controle de movimentos e à atenção a pessoas e objetos configurando mudança qualitativa na capacidade de interagir. Como discutem Mendes e Seidl-de-Moura (2015), nesse momento da ontogênese, bebês passam a coordenar ações comunicativas e a corresponder com olhares dirigidos, balbucios e sorrisos à fala e aos sorrisos maternos. Ainda nesse período, segundo as autoras, há evidências relatando a discriminação de certas emoções, a manutenção da atenção visual, a capacidade de imitação de expressões faciais e a apresentação de rudimentos de autorregulação, compondo um conjunto de habilidades que permitem e promovem trocas emocionais e afetivas. O surgimento do sorriso social como comportamento contingente a comportamentos afetivos maternos (Mendes, Seidl-de-Moura, 
\& Siqueira, 2009), bem como movimentos expressivos de boca, mãos e olho em presença de sorrisos, vocalizações e fala das mães, são indícios da capacidade de identificar, no outro, expressões faciais de emoção (Trevarthen, 2011).

Várias capacidades são adquiridas no segundo e terceiro meses de vida, com manifestações podendo variar em frequência e duração de acordo com o contexto sociocultural. Aos três meses, verificou-se que o bebê é capaz de reconhecer o cuidador, balbuciar, emitir sons dirigidos ao outro e se acalmar quando o outro fala com ele (Alvarenga, Paixão, Soares, \& Silva, 2018) e também discriminar os toques próprios dos procedentes de outra pessoa, facilitando a percepção de demonstrações afetivas, de acordo com Rochat (2007).

O reconhecimento, por parte da mãe, de preferências do(a) filho(a), de seus modos de se comunicar e reagir, vai se constituindo progressivamente. Tem sido abordado a partir do reconhecimento dos pedidos de atenção, das respostas afetivas, assim como também da fala materna dirigida ao bebê, o "manhês" (Arpini et al., 2015).

Com base no exposto e nas evidências trazidas, é cabível argumentar o quanto parece profícuo o aprofundamento dos estudos voltados para a dimensão emocional e afetiva do desenvolvimento inicial, manifestada no bojo das interações mãe-bebê. Desse modo, e ao considerar as interações iniciais e os aspectos constitutivos das trocas afetivas, como os comportamentos afetivos maternos e expressões emocionais de bebês, parece propício o objetivo do presente estudo, que foi o de produzir um quadro referencial mapeando e analisando o que vem sendo estudado sobre trocas afetivas nas interações iniciais entre mães e bebês em período recente, de 2008 a 2018, de modo a contribuir para a continuada construção do conhecimento sobre o desenvolvimento emocional e favorecendo o delineamento e definições de novas pesquisas a partir das lacunas encontradas.

\section{Método}

Realizou-se uma revisão integrativa da literatura, método indicado por Creswell (2007) para se organizar uma síntese de conhecimentos, em que foram seguidas cinco etapas: a) identificação do tema e questão para a elaboração da revisão integrativa; b) estabelecimento de critérios para inclusão e exclusão de estudos/amostragem ou busca na literatura; c) definição das informações a serem extraídas dos estudos selecionados/categorização dos estudos; d) avaliação dos estudos incluídos na revisão integrativa; e) apresentação da revisão/síntese do conhecimento. 
A busca foi realizada no primeiro trimestre de 2019, e foram consultadas as bases de dados Web of Science, PsycNet, PUBMED, Scielo Brasil e LILACS. Como expressão de busca, foram usados os termos mother, infant e affective exchanges e o boleano AND. Na língua portuguesa, foram utilizados os termos "mãe", "bebêe" e "trocas afetivas" com conector "e" (quando a sintaxe de busca da base de dados permitia). Foram estabelecidos como critérios de inclusão: (a) estudos empíricos voltados para interações mãe-bebê envolvendo afetividade e/ou reações emocionais de um deles ao outro; (b) publicados entre 2008 e 2018 (inclusive). Como critérios de exclusão, foi definido não selecionar: (a) ensaios clínicos, artigos de revisões sistemáticas e estudos teóricos, adaptação e avaliação de instrumentos, capítulos de livros; (b) estudos com participantes que não incluíssem bebês ou que investigassem bebês com quadro psicopatológico; (c) estudos publicados em outros idiomas que não fossem o português e o inglês.

A busca realizada identificou um total de 57 artigos. Retirados os 22 duplicados, foram lidos os títulos e resumos dos artigos restantes e, após a aplicação dos critérios de exclusão e inclusão, foram selecionados 17 artigos que seguiram para a etapa de leitura completa, procedendo-se a seguir a análise deles. A Figura 1 apresenta o fluxograma do processo de seleção dos artigos. 

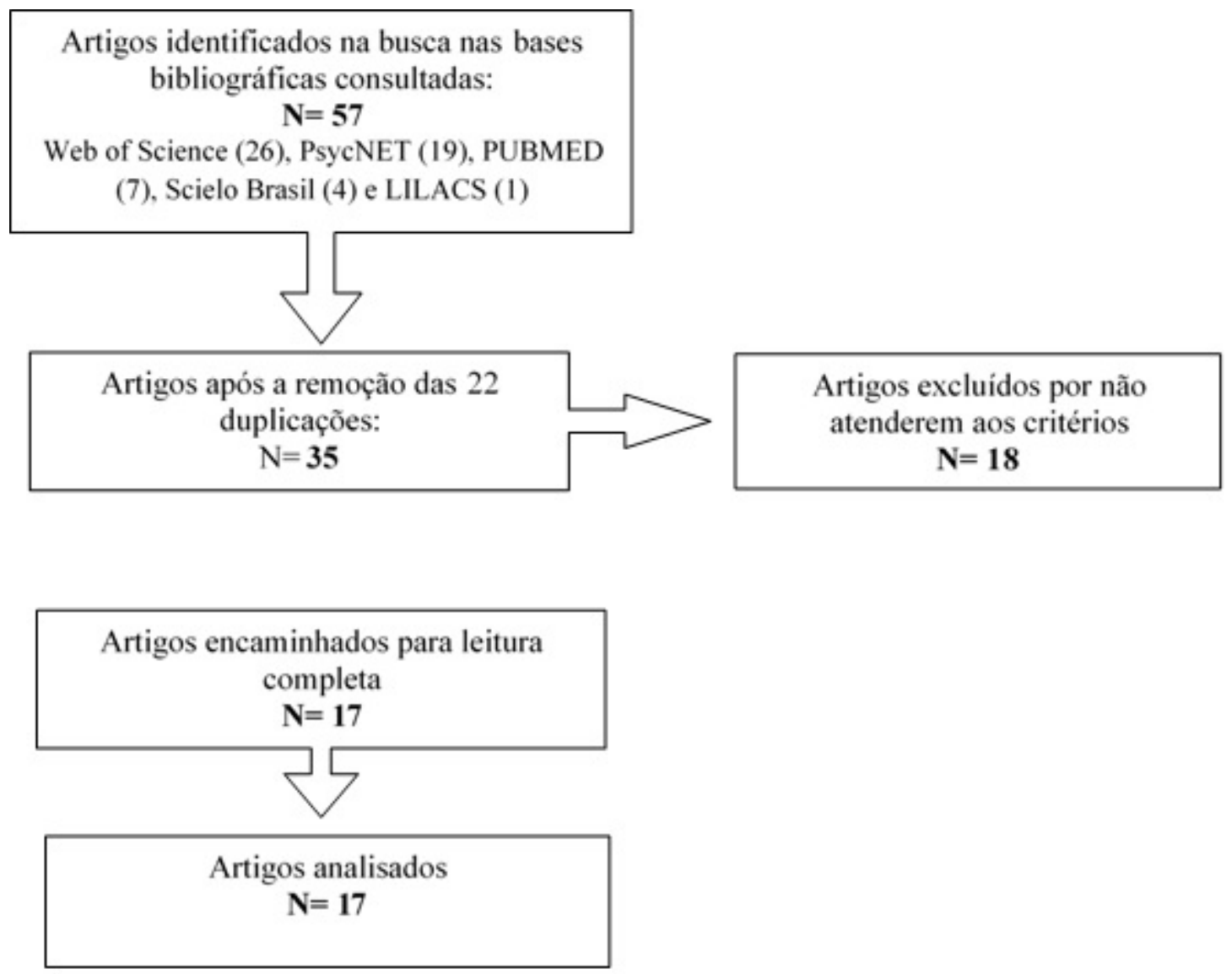

Figura 1. Fluxograma do processo de seleção dos artigos

Para a análise dos 17 artigos selecionados, foram definidas as seguintes categorias: ano de publicação (para verificar período de concentração das publicações sobre o tema); local de realização do estudo (cidade/país em que foi realizado o estudo, de modo a verificar se há concentração de publicações em um determinado país); participantes (para caracterização dos participantes e do contexto sociocultural em que viviam, e faixa etária do bebê); delineamento da pesquisa (se transversal ou longitudinal); técnicas de coleta de dados (para analisar as diversas técnicas empregadas na coleta de dados, como observações, questionários, entrevistas, tarefas, escalas ou combinação entre elas); temáticas e objetivos de estudo (para verificar aspectos centrais da temática geral abordados, tendo-se trabalhado com as seguintes categorias: dinâmica e circunstâncias das trocas afetivas iniciais; impactos da prematuridade; impactos da depressão materna; relações entre trocas afetivas e domínios do desenvolvimento). Tais categorias foram definidas pelas autoras, de modo independente, com base nos trechos dos estudos em que eram explicitados os objetivos. Caso tivessem ocorrido discordâncias, deveriam ser levadas para discussão no grupo de pesquisa de que fazem parte as autoras. 


\section{Resultados}

Analisando o ano de publicação dos artigos, observou-se que o ano que apresentou o maior número de publicações foi 2018, com cinco artigos. Com relação ao local de realização do estudo, uma parcela expressiva $(\mathrm{n}=10,59 \%)$ foi realizada em cidades de grande porte, como Londres (Fantasia, Costall, Fasulo, \& López, 2014), Miami (Hummel, Kiel, \& Zvirblyte, 2016), Nova York (Beebe et al., 2010; Beebe et al., 2018), Paris (Bydlowski, Apter, Golse, Lallane, \& Vaivre-Douret, 2015; Gratier \& Trevarthen, 2008), Rio de Janeiro (Seidl-de-Moura et al., 2008), Roma (Tambelli, Lucarelli, \& Odorisio, 2014), Salvador (Alvarenga et al., 2018), Santiago (Santelices, Olhaberry, Pérez-Salas, \& Carvacho, 2010) e Taipei (Taiwan) (Hsu \& Jeng, 2008). Dois foram realizados em cidades de porte médio, Santa Maria, Rio Grande do Sul, Brasil (Hoogstraten, Souza, \& Moraes, 2018) e Catalão, Goiás, Brasil (Souza \& Lemos, 2018). Quatro estudos não mencionaram a cidade/país em que foram realizados: Bourvis et al. (2018); Fabrizi, Costa, Lucarelli e Patruno (2010); Simonelli, Palo e Bighin (2014); Wang, Biringen e Morgan (2014).

Com relação à categoria participantes, a maioria dos estudos $(n=15,88 \%)$ colheu dados especificamente com mães e bebês e duas investigações analisaram também interações com outros perfis de parceiros, como pais, irmãos e educadores de creche (Santelices et al., 2010; Simonelli et al., 2014). Simonelli et al. (2014) apresentaram um quadro pouco estudado na literatura e significativo, sobre interações com ambos os pais e cooperação parental desde a gravidez. Verificou-se que somente quatro estudos (Beebe et al., 2018; Bourvis et al., 2018; Hoogstraten et al., 2018; Wang et al., 2014) analisaram e discutiram a diferença de sexo dos bebês. Quanto à faixa etária dos bebês, em dez estudos participaram mães e bebês de zero a seis meses (Alvarenga et al., 2018; Beebe et al., 2018; Bourvis et al., 2018; Bydlowski et al., 2015; Fantasia et al., 2014; Gratier \& Trevarthen, 2008; Hsu \& Jeng, 2008; Seidl-de-Moura et al., 2008; Souza \& Lemos, 2018; Tambelli et al., 2014). Três estudos compararam interações ocorridas em mais de um período do primeiro ano de vida: Beebe et al. (2010), quatro e doze meses; Hoogstraten et al. (2018) (3-4 meses; 6-7 meses e 29 dias; 8-9 meses e 29 dias); Simonelli et al. (2014) (quatro e nove meses). Quatro estudos analisaram bebês além do primeiro ano de vida: Santelices et al. (2010) (entre oito e 24 meses), Fabrizi et al. (2010) ( $M=33$ meses), Hummel et al. (2016) (24 meses) e Wang et al. (2014) (18 meses e 36 meses).

Em todos os 17 estudos, a população vivia em contextos urbanos, sendo que onze estudos (Alvarenga et al., 2018; Beebe et al., 2010; Fantasia et al., 2014; Hoogstraten et al., 2018; Hsu \& Jeng, 2008; Hummell et al., 2016; Santelices et al., 2010; Seidl-de-Moura et al., 
2008; Simonelli et al., 2014; Souza \& Lemos, 2018; Wang et al., 2014) apresentaram características sociodemográficas de seus participantes. Beebe et al. (2018), Bourvis et al. (2018), Bydlowski et al. (2015), Gratier e Trevarthen (2008), Fabrizi et al. (2010) e Tambelli et al. (2014) não caracterizaram claramente seus participantes quanto a tais aspectos.

Sobre o delineamento da pesquisa, dez estudos foram transversais (Alvarenga et al., 2018; Bourvis et al., 2018; Fabrizi et al., 2010; Fantasia et al., 2014; Gratier \& Trevarthen, 2008; Hummel et al., 2016; Hsu \& Jeng, 2008; Santelices et al., 2010; Seidl-de-Moura et al., 2008; Tambelli et al., 2014). Os estudos com delineamento longitudinal foram sete (Beebe et al., 2010; Beebe et al., 2018; Bydlowski et al., 2015; Hoogstraten et al., 2018; Simonelli et al., 2014; Souza \& Lemos, 2018; Wang et al., 2014).

Com relação às técnicas de coleta de dados, nove estudos utilizaram observações naturalísticas em diversos ambientes. Bydlowski et al. (2015), Fabrizi et al. (2010), Hoogstraten et al. (2018) e Tambelli et al. (2014) observaram bebês em unidades públicas de saúde; Alvarenga et al. (2018), Gratier e Trevarthen (2008), Souza e Lemos (2018) e Seidl-deMoura et al. (2008) em domicílios e Santelices et al. (2010) em domicílios e em creches. Verificou-se que dos nove estudos que realizaram observações naturalísticas, somente Seidlde-Moura et al. (2008) analisaram sequências de respostas em contexto de trocas afetivas mãe-bebê.

Oito estudos utilizaram observações em situações experimentais. Beebe et al. (2010), Bourvis et al. (2018), Hsu e Jeng (2008), e Hummel et al. (2016) aplicaram o procedimento Situação Estranha, de M. Ainsworth. Situações experimentais com brincadeira foram utilizadas por Beebe et al. (2018), Fantasia et al. (2014), Simonelli et al. (2014) e Wang et al. (2014).

Quanto ao uso de outras técnicas para coletar dados, Hummel et al. (2016) utilizaram uma tarefa de brincadeira e Wang et al. (2014), a tarefa Play-Doh. Cinco estudos utilizaram questionários respondidos pelas mães: Alvarenga et al. (2018), Fabrizi et al. (2010), Hummel et al. (2016), Tambelli et al. (2014) e Wang et al. (2014). Três estudos realizaram entrevistas com as mães: Bydlowsky et al. (2015), Hoogstraten et al. (2018) e Tambelli et al. (2014). Identificou-se o uso de escalas por Alvarenga et al. (2018), Bydlowski et al. (2015), Fabrizi et al. (2010), Fantasia et al. (2014); Hoogstraten et al. (2018); Hummel et al. (2016), Simonelli et al. (2014) e Wang et al. (2014).

Com relação à categoria temáticas estudadas e objetivos de estudo, verificou-se que os artigos selecionados se distribuíram do seguinte modo: 
- dinâmica e circunstâncias das trocas afetivas iniciais. Bourvis et al. (2018) consideraram a vocalização do bebê diante da retirada de expressividade materna no experimento de face imóvel como atitude ativa e tentativa de retomar trocas afetivas interrompidas, diferindo de Fantasia et al. (2014) que consideraram as reações dos bebês à retirada da expressividade materna como somente reação ao estresse provocado pelo experimento, sem considerar a tentativa de troca afetiva como atitude ativa. Beebe et al. (2010) tiveram como objetivo analisar as formas com que mãe e bebê regulam em conjunto atenção, orientação espacial e toque nas interações face a face aos quatro meses e a influência da qualidade dessas trocas afetivas ocorridas, na natureza do vínculo aos 12 meses. Disponibilidade emocional, sensibilidade e adaptação maternas ao bebê como elementos para o estabelecimento de trocas afetivas foram discutidas por Souza e Lemos (2018). Atividades de musicalização como possibilidade de estabelecimento de trocas afetivas mãe-bebê foram abordadas por Gratier e Trevarthen (2008). A cooperação parental como ferramenta para o suporte de cuidados ao bebê foi discutida por Simonelli et al. (2014). Santelices et al. (2010) abordaram a diversidade de perfis de parceiros adultos, educadores e cuidadores primários, na interação com bebês, temática que tem sido pouco contemplada nos estudos encontrados na literatura. Wang et al. (2014) consideraram que o desenvolvimento da autonomia, da persistência e de competências nas crianças têm raízes profundas nas interações iniciais mãebebê. Seidl-de-Moura et al. (2008) analisaram os comportamentos dos parceiros, a complexidade das trocas e seu componente afetivo em interações mãe-bebê. No entanto, não foi verificada nesse estudo a relação entre os comportamentos afetivos maternos e as expressões emocionais de bebês em termos de reciprocidade afetiva.

- impactos da prematuridade. A importância do toque afetivo, do olhar e da fala dirigida do adulto para que o bebê recém-nascido prematuro possa, por meio da conexão emocional, desenvolver suas predisposições herdadas para interagir e realizar trocas com o adulto, foi discutida em Beebe et al. (2018). A comparação da sensibilidade e capacidade de autorregulação entre bebês a termo e prematuros diante da retirada das expressões maternas de afeto foi avaliada por Hsu e Jeng (2008). Os autores verificaram que as capacidades de atenção e de dar respostas afetivas, de bebês nascidos a termo e de prematuros saudáveis, às mudanças nas expressões faciais maternas, podem ser semelhantes aos dois meses de idade.

- impactos da depressão materna. Bydlowsky et al. (2015), Hummel et al. (2016) e Tambelli et al. (2014) analisaram o impacto da depressão materna nas trocas afetivas mãebebê, sugerindo que disponibilidade emocional e comportamentos afetivos maternos dirigidos ao bebê sejam vitais para o estabelecimento de trocas afetivas ao longo do desenvolvimento. 
Bydlowsky et al. (2015) relacionaram níveis de depressão materna pós-parto com a capacidade de autorregulação de bebês, sobretudo com o reflexo mão-boca. Hummel et al. (2016) abordaram a redução da afetividade e do envolvimento materno com o bebê como um prejuízo nas trocas afetivas relacionado a sintomas depressivos maternos. Tambelli et al. (2014) discutiram a influência da depressão nos padrões interacionais mãe-bebê e nas representações que as mães têm sobre as suas relações com bebês. Alvarenga et al. (2018) avaliaram efeitos da depressão materna sobre a frequência de sorrisos maternos, de estimulação tátil e de estimulação com objetos, nas interações com bebês. Nesse estudo, as evidências do impacto da depressão pós-parto sobre os comportamentos sensíveis da mãe e do impacto da estimulação não-verbal materna (e da falta dessa estimulação) sobre o desenvolvimento motor do bebê foram consideradas, mas as sequências que caracterizam trocas afetivas com as expressões emocionais dos bebês não foram priorizadas.

- relações entre trocas afetivas e domínios do desenvolvimento. Fabrizi et al. (2010) abordaram a qualidade das trocas afetivas mãe-criança e sua relação com as alterações no desenvolvimento da linguagem da criança, abordando ainda o isolamento e a recusa alimentar. Hoogstraten et al. (2018) discutiram a presença de indicadores de desenvolvimento, como sorrisos e vocalizações do bebê, em resposta à mãe ou a quem está se dirigindo a ele e a presença de comportamentos maternos dirigidos ao bebê, especialmente sorrisos e fala, como indicadores de desenvolvimento saudável.

Ao analisar o conjunto dos artigos quanto à temática estudada e ao objetivo do estudo, observou-se também que onze estudos não tiveram como objetivo observar sequências de respostas e interações entre comportamentos afetivos maternos e expressões emocionais de bebês. Beebe et al. (2018), Bourvis et al. (2018), Fantasia et al. (2014), Hsu e Jeng (2008), Simonelli et al. (2014) e Wang et al. (2014) priorizaram análises das reações de mães e de bebês em uma situação experimental padronizada. Diferentemente, Hummel et al. (2016) e Beebe et al. (2010) compararam sequências de respostas em contexto de trocas afetivas mãebebê, mas, priorizaram análises de comportamentos afetivos maternos e sua influência nas expressões emocionais do bebê (análise da contingência materna para caracterização do estilo de apego em Beebe et al., 2010; caracterização de trocas afetivas relacionadas à presença ou ausência de sintomas depressivos maternos em Hummel et al., 2016) sem discutir a influência das expressões emocionais dos bebês nas trocas afetivas, mesmo tendo estabelecido categorias de análise para tal. Outros estudos, como Alvarenga et al. (2018), Souza e Lemos (2018) e Hoogstraten et al. (2018), também priorizaram a análise de comportamentos afetivos maternos, deixando de analisar as sequências que caracterizariam trocas afetivas. 
Na Tabela 1, são apresentadas as categorias pré-estabelecidas para a análise dos artigos selecionados, configurando as características de interesse para apreciação dos estudos. 
Tabela 1

Sumário das características dos estudos

\begin{tabular}{|c|c|c|c|c|c|c|}
\hline $\begin{array}{l}\text { Estudo Ano } \\
\text { publicação }\end{array}$ & $\begin{array}{l}\text { Local do } \\
\text { Estudo }\end{array}$ & Participantes & Delineamento & $\begin{array}{l}\text { Técnica } \\
\text { Coleta }\end{array}$ & $\begin{array}{l}\text { Temátical } \\
\text { objetivo }\end{array}$ & Principais resul tados \\
\hline $\begin{array}{l}\text { Alvarenga et al. } \\
(2018)\end{array}$ & $\begin{array}{l}\text { Salvador } \\
\text { Brasil }\end{array}$ & Maes/bebês (3 m) & $\mathrm{T}$ & Escala; questionário & $\begin{array}{l}\text { Impactos de } \\
\text { depressão materna }\end{array}$ & $\begin{array}{l}\text { Depressão ma terna diminui frequência de } \\
\text { sorrisos da mãe, impactando as interações } \\
\text { com o bebê. }\end{array}$ \\
\hline Beebe et al. (2010) & $\begin{array}{l}\text { Nova Iorque } \\
\text { EUA }\end{array}$ & $\begin{array}{l}\text { Maes-bebês } \\
(4 \text { e } 12 \mathrm{~m})\end{array}$ & L & $\begin{array}{l}\text { Sit. experimental } \\
\text { brincadeira }\end{array}$ & $\begin{array}{l}\text { Dinâmica e } \\
\text { circunstâncias } \\
\text { trocas afetivas } \\
\text { iniciais }\end{array}$ & $\begin{array}{l}\text { Contingência de comportamentos } \\
\text { afe tivos maternos impacta } \\
\text { esta belecimento e estilo de apego do } \\
\text { be bê. }\end{array}$ \\
\hline Beebe et al. (2018) & $\begin{array}{l}\text { Nova Iorque } \\
\text { EUA }\end{array}$ & Maes' bebês $(0 \mathrm{e} 4 \mathrm{~m})$ & L & $\begin{array}{l}\text { Observações e Sit. } \\
\text { experimental. } \\
\text { brincadeira }\end{array}$ & $\begin{array}{l}\text { Impactos da } \\
\text { prematuridade }\end{array}$ & $\begin{array}{l}\text { Toque afetivo materno em prematuros } \\
\text { favorece trocas afetivas mãe-bebê. }\end{array}$ \\
\hline $\begin{array}{l}\text { Bourvis et al. } \\
(2018)\end{array}$ & Não informado & Maes-bebês ( 3 e $6 \mathrm{~m}$ ) & $\mathrm{T}$ & $\begin{array}{l}\text { Sit. experimental } \\
\text { brincadeira }\end{array}$ & $\begin{array}{l}\text { Dinâmica e } \\
\text { circunstâncias. } \\
\text { trocas afetivas } \\
\text { iniciais }\end{array}$ & $\begin{array}{l}\text { Importância de considerar vocalizações } \\
\text { dos bebês como atitude ativa e tentativa } \\
\text { de retomar trocas afetivas. }\end{array}$ \\
\hline $\begin{array}{l}\text { Bydlowski et al. } \\
\text { (2015) }\end{array}$ & $\begin{array}{l}\text { Paris } \\
\text { França }\end{array}$ & $\begin{array}{l}\text { Maes-bebês } \\
(0 \text { e } 2 \mathrm{~m})\end{array}$ & L & $\begin{array}{l}\text { Entrevistas; } \\
\text { observações } \\
\text { naturalisticas. } \\
\text { escala }\end{array}$ & $\begin{array}{l}\text { Impactos de } \\
\text { depressão materna }\end{array}$ & $\begin{array}{l}\text { Comportamentos afetivos maternos } \\
\text { facilitam coordenação mão/boca de bebês } \\
\text { de } 0 \text { a } 2 \text { meses. }\end{array}$ \\
\hline $\begin{array}{l}\text { Fabrizi et al. } \\
(2010)\end{array}$ & Itália & $\begin{array}{c}\text { Maes-cri } \\
\text { (33m em média) }\end{array}$ & $\mathrm{T}$ & $\begin{array}{l}\text { Escalas; } \\
\text { questionários }\end{array}$ & $\begin{array}{l}\text { Rel. trocas afetivas } \\
\text { e dominios } \\
\text { de senvolvimento. }\end{array}$ & $\begin{array}{l}\text { Trocas afetivas iniciais têm efeitos no } \\
\text { de senvolvimento da linguagem e na } \\
\text { alimentação. }\end{array}$ \\
\hline $\begin{array}{l}\text { Fanta sia et al. } \\
(2014)\end{array}$ & Londres Inglaterra & Maes' bebês ( 0 a $3 \mathrm{~m}$ ) & $\mathrm{T}$ & $\begin{array}{l}\text { Sit. experimental } \\
\text { brincadeira }\end{array}$ & $\begin{array}{l}\text { Dinâmica e circ. } \\
\text { trocas afetivas } \\
\text { iniciais }\end{array}$ & $\begin{array}{l}\text { Interruppão de trocas afetivas, com } \\
\text { supressão de fala e gestos maternos (em } \\
\text { situação experimental), provoca estresse } \\
\text { no bebê. }\end{array}$ \\
\hline $\begin{array}{l}\text { Gratier e } \\
\text { Trevarthen (2008) }\end{array}$ & $\begin{array}{c}\text { Paris } \\
\text { França }\end{array}$ & $\begin{array}{l}\text { Mae-bebê; } \\
\quad(2 \mathrm{~m})\end{array}$ & $\mathrm{T}$ & $\begin{array}{l}\text { Observações } \\
\text { naturalisticas }\end{array}$ & $\begin{array}{l}\text { Dinâmica e } \\
\text { circunstâncias. } \\
\text { trocas afetivas } \\
\text { iniciais }\end{array}$ & $\begin{array}{l}\text { Atividades de musicalização favorecem } \\
\text { estabelecimento de trocas afetivas mãe- } \\
\text { bebê, em bebês de dois meses. }\end{array}$ \\
\hline $\begin{array}{l}\text { Hoogstraten et al. } \\
\text { (2018) }\end{array}$ & Santa Maria Brasi1 & Maes-bebês (3 a $9 \mathrm{~m}$ ) & $\mathrm{L}$ & $\begin{array}{l}\text { Observações; } \\
\text { entrevistas }\end{array}$ & $\begin{array}{l}\text { Rel. trocas afetivas } \\
\text { e dominios } \\
\text { desenvolvimento. }\end{array}$ & $\begin{array}{l}\text { Fatores psicossociais associados à } \\
\text { presença de alterações em indicadores de } \\
\text { no de senvolvimento tipico indicaram } \\
\text { risco psiquico. }\end{array}$ \\
\hline Hsu e Jeng (2008) & $\begin{array}{l}\text { Taipei } \\
\text { Taiwan }\end{array}$ & $\begin{array}{l}\text { Mies-bebês; } \\
\quad(2 \mathrm{~m})\end{array}$ & $\mathrm{T}$ & $\begin{array}{l}\text { Sit. experimental } \\
\text { brincadeira }\end{array}$ & $\begin{array}{l}\text { Impactos da } \\
\text { prematuridade }\end{array}$ & $\begin{array}{l}\text { Não houve diferenças entre prematuros e } \\
\text { na scidos a termo, quanto à } \\
\text { sensibilidade/regulação emocional, diante } \\
\text { da supressão de expressões maternas de } \\
\text { afe to. }\end{array}$ \\
\hline $\begin{array}{l}\text { Hummel et al. } \\
\text { (2016) }\end{array}$ & $\begin{array}{l}\text { Miami } \\
\text { EUA }\end{array}$ & $\begin{array}{l}\text { Maes-bebês } \\
(24 \mathrm{~m})\end{array}$ & $\mathrm{T}$ & $\begin{array}{l}\text { Tarefa; escala; } \\
\text { questionário }\end{array}$ & $\begin{array}{l}\text { Impactos de } \\
\text { depressão materna. }\end{array}$ & $\begin{array}{l}\text { Redução da afetividade e do } \\
\text { envolvimento materno associadas a } \\
\text { prejuizo nas trocas afetivas com bebê. }\end{array}$ \\
\hline $\begin{array}{l}\text { Santelices et al. } \\
(2010)\end{array}$ & $\begin{array}{l}\text { Santiago } \\
\text { Chile }\end{array}$ & $\begin{array}{l}\text { Maies; pais; irmãos; } \\
\text { educadores } \\
\text { (8 a } 24 \text { m) }\end{array}$ & $\mathrm{T}$ & $\begin{array}{l}\text { Observações } \\
\text { naturalisticas }\end{array}$ & $\begin{array}{l}\text { Dinâmica e } \\
\text { circunstâncias. } \\
\text { trocas afetivas } \\
\text { iniciais }\end{array}$ & $\begin{array}{l}\text { Diferenças de ênfase em aspectos } \\
\text { comunica tivos e cognitivos nas interações } \\
\text { em domicilio e na creche. }\end{array}$ \\
\hline $\begin{array}{l}\text { Seidl-de-Moura et } \\
\text { al. (2008) }\end{array}$ & $\begin{array}{l}\text { Rio de Janeiro } \\
\text { Brasil }\end{array}$ & $\begin{array}{l}\text { Mies-bebês; } \\
(0 \text { e } 5 \mathrm{~m})\end{array}$ & $\mathrm{T}$ & $\begin{array}{l}\text { Observações } \\
\text { naturalisticas }\end{array}$ & $\begin{array}{l}\text { Dinâmica e } \\
\text { circunstâncias. } \\
\text { trocas afetivas } \\
\text { iniciais }\end{array}$ & $\begin{array}{l}\text { Efeito da idade do bebê na complexidade } \\
\text { dasinterações com a mãe e na } \\
\text { manifestação de afetividade reciproca dos } \\
\text { parceiros. }\end{array}$ \\
\hline $\begin{array}{l}\text { Simonelli et al. } \\
\text { (2014) }\end{array}$ & Itảlia & $\begin{array}{l}\text { Maes-bebês } \\
(4 \text { e } 9 \mathrm{~m})\end{array}$ & L & $\begin{array}{l}\text { Sit. experimental } \\
\text { brincadeira; } \\
\text { questionário }\end{array}$ & $\begin{array}{l}\text { Dinâmica e } \\
\text { circunstâncias. } \\
\text { trocas afetivas } \\
\text { iniciais }\end{array}$ & $\begin{array}{l}\text { Crescimento das interações familiares é } \\
\text { relacionado ao papel ativo do bebê nessas } \\
\text { interações. }\end{array}$ \\
\hline $\begin{array}{l}\text { Souza e Lemos } \\
\text { (2018) }\end{array}$ & Catalão Brasil & $\begin{array}{l}\text { Mae-bebê } \\
(0 \text { a } 2 \mathrm{~m})\end{array}$ & $\mathrm{L}$ & $\begin{array}{l}\text { Observações } \\
\text { naturalisticas }\end{array}$ & $\begin{array}{l}\text { Dinâmica e } \\
\text { circunstâncias } \\
\text { trocas afetivas } \\
\text { iniciais }\end{array}$ & $\begin{array}{l}\text { Disponibilidade emocional, sensibilidade } \\
\text { e adaptação maternas ao bebê são } \\
\text { essenciais para o estabelecimento de } \\
\text { trocas afetivas. }\end{array}$ \\
\hline $\begin{array}{l}\text { Tambelli et al. } \\
\text { (2014) }\end{array}$ & $\begin{array}{l}\text { Roma } \\
\text { Itália }\end{array}$ & $\begin{array}{l}\text { Maes-bebês } \\
(4 \mathrm{~m})\end{array}$ & $\mathrm{T}$ & $\begin{array}{l}\text { Entrevistas; } \\
\text { observações; } \\
\text { questionários }\end{array}$ & $\begin{array}{l}\text { Impactos de } \\
\text { depressão materna }\end{array}$ & $\begin{array}{l}\text { Bebés demonstraram trocas } \\
\text { comunicativas conflitivas e recusa } \\
\text { alimentar em grupo de mães com } \\
\text { depressão. }\end{array}$ \\
\hline Wang et al. (2014) & Não informado & $\begin{array}{l}\text { Meses-bebês; } \\
(18 \text { e } 36 \mathrm{~m})\end{array}$ & $\mathrm{L}$ & $\begin{array}{l}\text { Sit. experimental } \\
\text { brincadeira } \\
\text { entrevista; } \\
\text { questionário }\end{array}$ & $\begin{array}{l}\text { Dinâmica e } \\
\text { circunstâncias. } \\
\text { trocas afetivas } \\
\text { iniciais }\end{array}$ & $\begin{array}{l}\text { Trocas afetivas iniciais mãe-bebê } \\
\text { facilitam a aquisição de autonomia nas } \\
\text { crianças. }\end{array}$ \\
\hline
\end{tabular}




\section{Discussão}

O presente estudo teve como objetivo realizar uma revisão integrativa da literatura, abrangendo período recente (2008-2018), de estudos empíricos sobre trocas afetivas mãebebê, visando contribuir para o conhecimento do que vem sendo produzido nessa temática, identificando e analisando os estudos de acordo com suas características, segundo categorias predefinidas.

Primeiramente, chamou a atenção o pequeno número de estudos encontrados sobre a temática, dentro das especificidades dessa revisão de literatura (17 estudos selecionados), em um período recente, indicando a carência de novos estudos sobre o tema. A totalidade das investigações ter sido realizada em contextos urbanos indica uma lacuna no que respeita ao estudo de contextos não-urbanos. De acordo com Henrich, Heine e Norenzayan (2010), boa parte dos estudos de Psicologia envolve participantes que vivem em ambientes urbanos e em cidades de grande porte, sinalizando a necessidade de pesquisas a serem realizadas em contextos diversos.

A comparação feita por Santelices et al. (2010) sobre interações afetivas entre cuidadores primários e educadores de creche traz importantes informações sobre a diversidade de perfis de parceiros adultos em trocas afetivas com bebês. A participação do pai em conjunto com a mãe e seus efeitos nas trocas afetivas com as crianças, em Simonelli et al. (2014), apresenta um enfoque diferenciado e de relevância para se pensar o desenvolvimento emocional dos bebês. Muito embora os termos de busca tenham promovido a obtenção de estudos envolvendo necessariamente a mãe, pode ser sublinhado o número reduzido de estudos em que o pai também foi participante.

Verificou-se que em um número significativo de estudos, os participantes foram mães e bebês de zero a seis meses (Alvarenga et al., 2018; Beebe et al., 2018; Bourvis et al., 2018; Bydlowski et al., 2015; Fantasia et al., 2014; Gratier \& Trevarthen, 2008; Hsu \& Jeng, 2008; Seidl-de-Moura et al., 2008; Souza \& Lemos, 2018; Tambelli et al., 2014), enfatizando a importância de: (1) estabelecimento do vínculo e das trocas afetivas iniciais no desenvolvimento; (2) de conhecer-se melhor sobre comportamentos afetivos maternos nas interações iniciais e seus possíveis efeitos posteriores no desenvolvimento; e (3) a ativa participação de bebês na restauração de rupturas na comunicação com suas mães. Os estudos que compararam interações ocorridas em mais de um período do primeiro ano de vida (Beebe et al., 2010; Hoogstraten et al., 2018; Simonelli et al., 2014) e os estudos que analisaram bebês além do primeiro ano de vida (Santelices et al., 2010; Fabrizi et al., 2010; Hummel et 
al., 2016; Wang et al., 2014), priorizaram a análise de impactos das trocas afetivas iniciais em períodos posteriores do desenvolvimento.

Quanto às técnicas de coleta de dados, foram variadas. Um número significativo de estudos colheu dados por meio de observações sistemáticas e outros estudos utilizaram relatos dos participantes por entrevistas, escalas, questionários e situações experimentais associadas a métodos de observação. Nenhum instrumento se mostrou mais amplamente usado nos estudos encontrados nesta revisão, configurando não haver priorização por parte dos pesquisadores nesse campo de investigação por algum instrumento específico.

Quanto a temáticas estudadas e objetivos, verificou-se que foram predominantes os estudos com populações em desenvolvimento típico (resultado possivelmente relacionado ao critério de exclusão de não selecionar estudos que investigassem bebês com quadro psicopatológico), mas havendo também estudos com prematuros e sobre interações iniciais de bebês com mães em situação de depressão pós-parto. Poucos estudos analisaram sequências de comportamentos afetivos de mães em resposta a expressões emocionais de bebês e viceversa, o que permitiria caracterizar as modificações, em termos de morfologia e frequência, nas expressões emocionais do bebê dirigidas à mãe, tanto respondendo como desencadeando comportamentos afetivos maternos e os comportamentos afetivos maternos dirigidos ao bebê, configurando um panorama de suma importância para a análise de trocas afetivas entre esses parceiros.

\section{Considerações Finais}

Este estudo produziu um quadro referencial, mapeando e analisando o que vem sendo estudado sobre trocas afetivas entre mães e bebês nas interações iniciais, em período recente, contribuindo com reflexões sobre o panorama das pesquisas na área. Verificou-se reduzida produção científica sobre essa temática, em que se aponta especialmente a necessidade de novos estudos brasileiros.

Como todos os trabalhos ocorreram em ambiente urbano, alerta-se para a relevância da realização de pesquisas em contextos não-urbanos. A maioria dos estudos encontrados ocupou-se de trocas apenas entre mães e bebês (em parte atribuível a viés da busca que fixou os termos "mãe", "bebê" e "trocas afetivas"), indicando uma oportuna ampliação das pesquisas de forma que incluam pais, outros cuidadores e educadores. Outro aspecto a ser observado se refere ao baixo número de estudos que analisaram e discutiram a diferença de sexo dos bebês nas trocas afetivas, sinalizando haver essa lacuna. Salienta-se ainda a carência 
de estudos que analisem sequências de comportamentos afetivos de mães em resposta a expressões emocionais de bebês e vice-versa, uma vez que investigações com esse foco de interesses proveem informações cruciais à compreensão dos processos iniciais de desenvolvimento emocional e afetivo.

Dentre as limitações desse estudo, considera-se que o envolvimento de outras bases de dados, outros idiomas e o emprego de diferentes estratégias de busca (utilizando outras combinações de termos) poderia ser proveitoso no sentido de ampliar o número de artigos selecionados e o potencial de análise. Ainda assim, e uma vez que se pretendeu mapear e discutir o cenário de estudos sobre trocas afetivas mãe-bebê, entende-se que o presente trabalho contribuiu para o esquadrinhamento dos principais aspectos que vêm sendo investigados a respeito destas trocas e das opções de método empregadas, bem como para a identificação de lacunas na área. Assim sendo, acredita-se que os objetivos estabelecidos tenham sido atingidos, favorecendo investigações futuras.

\section{Referências}

Alvarenga, P., Paixão, C., Soares, Z. F., \& Silva, A. C. S. (2018). Impacto da saúde mental materna na interação mãe-bebê e seus efeitos sobre o desenvolvimento infantil. Psico, 49(3), 317-327. doi: 10.15448/1980-8623.2018.3.28475

Arpini, D. M., Zanatta, E., Marchesan, R. Q., Faraj, S. P., Ledur, C. S., \& Mozzaquatro, C. O. (2015). Interação mãe-bebê: Um processo de descobertas. Interação em Psicologia, 19(1), 1-11. doi: 10.5380/psi.v19i1.32503

Beebe, B., Andrews, H., Bahrick, L., Buck, K., Chen, H., Cohen, P. .. \& Markese, S. (2010). The Origins of 12-Month Attachment: A Microanalysis of Four-Month Mother-Infant Interaction. Attachment Human Development, 12(1-2), 3-141. doi: $10.1080 / 14616730903338985$

Beebe, B., Myers, M., Lee, S. H., Lance, A., Ewing, J., Rubinckik, N., Andrews, H. .... \& Welch, M. G. (2018). Family nurture intervention for preterm infants facilitates positive mother-infant face-to-face engagement at 4 months. Developmental Psychology, 54(11), 2016-2031. doi: 10.1037.dev0000557

Bourvis, N., Singer, M., Saint Georges, C., Bodeau, N., Chetouani, M., Cohen, D., ... \& Feldman, R. (2018). Pre-linguistic infants employ complex communicative loops to engage mothers in social exchanges and repair interaction ruptures. Royal Society Open Science, 5(1), 170274. doi: 10.1098/rsos.170274 
Bydlowski, S., Apter, G., Golse, B., Lalanne, C., \& Vaivre-Douret, L. (2015). Construction of the mother-infant interactions from birth to two months. Links with the postpartum blues. Neuropsychiatrie de l' Enfance et de l'Adolescence, 63(1), 1-8. doi: 10.1016/j.neurenf.2014.08.004

Cole, P., \& Moore, G. A. (2014). About Face! Infant Facial Expression of Emotion. Emotion Review, 7(2), 116-120. doi: 10.1177/1754073914554786

Creswell, J. W. (2007). Projeto de pesquisa: Métodos qualitativo, quantitativo e misto. Porto Alegre: Artmed.

Fabrizi, A., Costa, A., Lucarelli, A., \& Patruno, E. (2010). Comorbidity in specific language disorders and early feeding disorders: Mother-child interactive patterns. Eating Weight Disorder, 15, 152-160. doi: 10.1007/BF03325294

Fantasia, V., Fasulo, A., Costall, A., \& López, B. (2014). Changing the game: Infant's participation in early play routine. Frontiers in Psychology, 5, 1-9. doi: $10.3389 /$ fpsyg.2014.00522

Gratier, M., \& Trevarthen, C. (2008). Musical narrative and motives for culture in motherinfant vocal interaction. Journal of Consciousness Studies, 15(10-11), 122-158. Recuperado de https://psycnet.apa.org/record/2008-15300-005

Henrich, J., Heine, S. J., \& Norenzayan, A. (2010). The weirdest people in the world? Behavioral and Brain Sciences, 33(2-3), 61-135. doi: 10.1017/S0140525X0999152X

Hoogstraten, A. M. R. J., Souza, A. P. R., \& Moraes, A. B. (2018). Indicadores clínicos de referência ao desenvolvimento infantil e sua relação com fatores obstétricos, psicossociais e sociodemográficos. Saúde e Pesquisa, 11(3), 589-601. doi: 10.17765/1983-1870.2018v11n3p589-601

Hummel, A. C., Kiel, E. J., \& Zvirblyte, S. (2016). Bidirectional effects of positive affect, warmth, and interactions between mothers with and without syntoms of depression and their toddlers. Journal of Child and Family Studies, 25(3), 781-789. doi: $10.1007 / \mathrm{s} 10826-015-0272-\mathrm{x}$

Hsu, H., \& Jeng, S. F. (2008). Two-month-olds' attention and affective response to maternal still face: A comparison between term and preterm infants in Taiwan. Infant Behavior \& Development, 31(2), 194-206. doi: 10.1016/j.infbeh.2007.10.008

Mendes, D. M. L. F., Seidl-de-Moura, M. L., \& Siqueira, J. O. (2009). The ontogenesis of smiling and its association with mothers' affective behaviors: A longitudinal data. Infant Behavior and Development, 32(4), 445-453. doi: 10.1016/j.infbeh.2009.07.004 
Mendes, D. M. L. F, \& Seidl-de-Moura, M. L. (2015). Facial Expressions in mother-baby interactional contexts and emotional development. In B. Flores (Ed.), Baby interactional contexts and emotional developmental facial expressions: recognition, developmental differences and social importance (pp. 91-108). New York: Nova Science Publishers.

Rochat, P. (2007). Intentional action arises from early reciprocal exchanges. Acta Psychologica, 124(1), 8-25. doi: 10.1016/j.actpsy.2006.09.004

Rochat, P., \& Striano, T. (2010). Social-cognitive development in the first year. In P. Rochat (Eds.), Early Social Cognition: Understanding Others in the First Months of Life (pp. 3-34). New Jersey: Lawrence Erlbaum.

Santelices, M. P., Olhaberry, M., Pérez-Salas, C. P., \& Carvacho, C. (2010). Comparative study of early interactions in mother-child dyads and care centre staff-child within the context of Chilean crèches. Child: Care, Health and Development, 36(2), 255-264. doi: 10.1111/j.1365-2214.2009.01032.x

Seidl-de-Moura, M. L., \& Ribas, A. F. P. (2004). Evidências sobre características de bebês recém-nascidos: Um convite a reflexões teóricas. In M. L. Seidl-de-Moura (Org.), $O$ bebê do século XXI e a psicologia em desenvolvimento (pp. 21-59). São Paulo, SP: Casa do Psicólogo.

Seidl-de-Moura, M. L., Ribas, A. F. P., Seabra, K. C., Nogueira, S. E, Pessôa, L. F., Mendes, D. M. L. F., ... \& Vicente, C. C. (2008). Interações mãe-bebê de um e cinco meses: Aspectos afetivos, complexidade e sistemas parentais predominantes. Psicologia: Reflexão e Crítica, 21(1), 66-73. doi: 10.1590/s0102-79722008000100009

Simonelli, A., Palo, F. D., \& Bighin, M. (2014). From pregnancy to the 9th month: The development of early interactive-relational competencies of the child in the family context. Interdisciplinary Journal of Family Studies, 19(1), 110-130. doi: $10.13140 / 2.1 .4110 .1442$

Souza, J. C., \& Lemos, M. F. (2018). Observação da relação mãe-bebê nos primeiros meses de vida. Perspectivas em Psicologia, 22(1), 18-37. doi: 10.14393/PPv22n1a2018-03

Tambelli, R., Odorisio, F., \& Lucarelli, L. (2014). Prenatal and Postnatal Maternal Representations in nonrisk and at-risk parenting: Exploring the influences on motherinfant feeding interactions. Infant Mental Health Journal, 35(4), 376-388. doi: 10.1002/imhj.21448

Thompson, R. A. (2015). Doing it with feeling: The emotion in early socioemotional development. Emotion Review, 7(1), 1-5. doi: 10.1177/1754073914554777 
Trevarthen, C. (2011). What is like to be a person who knows nothing. Defining the active intersubjective mind of a newborn human being infant and child development. Infant and Child Development, 20(1), 119-135. doi: 10.1002/icd.689

Wang, J, Biringen, Z., \& Morgan, G. A. (2014). Mother-Toddler Affect Exchanges and Children's Mastery Behaviors during Preschool Years. Infant and Child Development, 23(2), 139-152. doi: 10.1002/icd.1825

Walton, G. E., Bower, N. J. A., \& Bower, T. G. R. (1992). Recognition of familiar faces by newborns. Infant Behavior and Development, 15(2), 265-269. doi: 10.1016/01636383(92)80027-R

\section{Endereço para correspondência}

\section{Clarice Bieler}

Rua Max Fleiuss, 14, Tijuca, Rio de Janeiro - RJ, Brasil. CEP 20530-280

Endereço eletrônico: cbieler95@gmail.com

\section{Deise Maria Leal Fernandes Mendes}

Rua Edwaldo de Vasconcellos 20 ap. 302, Recreio dos Bandeirantes, Rio de Janeiro - RJ, Brasil. CEP 22795-385 Endereço eletrônico: deisefmendes@gmail.com

Recebido em: 24/10/2019

Reformulado em: 10/03/2020

Aceito em: 27/04/2020

\section{Notas}

* Mestrado em Psicologia Social pela UERJ. Doutoranda em Psicologia Social na UERJ.

** Doutora em Psicologia Social pela UERJ. Professor Associado da UERJ, coordenadora do laboratório Desenvolvimento Socioemocional e Parentalidade (diretório do CNPq).

Este artigo de revista Estudos e Pesquisas em Psicologia é licenciado sob uma Licença Creative Commons Atribuição-Não Comercial 3.0 Não Adaptada. 